\title{
A kinetic model for curcumin production in Escherichia coli
}

\author{
Daniel Machado, Lígia R. Rodrigues, Isabel Rocha* \\ Centre of Biological Engineering, University of Minho, Campus de Gualtar, 4710-057 Braga, Portugal
}

\section{A R T I C L E I N F O}

\section{Article history:}

Received 12 March 2013

Received in revised form 1 August 2014

Accepted 1 September 2014

Available online 16 September 2014

\section{Keywords:}

Metabolic engineering

Kinetic modeling

Escherichia coli

\begin{abstract}
A B S T R A C T
Curcumin is a natural compound obtained from turmeric, and is well known for its pharmacological effects. In this work, we design a heterologous pathway for industrial production of curcumin in Escherichia coli. A kinetic model of the pathway is then developed and connected to a kinetic model of the central carbon metabolism of $E$. coli. This model is used for optimization of the mutant strain through a rational design approach, and two manipulation targets are identified for overexpression. Dynamic simulations are then performed to compare the curcumin production profiles of the different mutant strains. Our results show that it is possible to obtain a significant improvement in the curcumin production rates with the proposed mutants. The kinetic model here developed can be an important framework to optimize curcumin production at an industrial scale and add value to its biomedical potential.
\end{abstract}

(c) 2014 Elsevier Ireland Ltd. All rights reserved.

\section{Introduction}

Curcumin is the yellow pigment from turmeric, a well known culinary spice obtained from the herb Curcuma longa. Research has shown that curcumin presents a wide range of pharmacological effects, including anti-inflammatory, anti-oxidant and anticarcinogenic activity (Aggarwal et al., 2003; D’Aguanno et al., 2012; Palve and Nayak, 2012). Given its therapeutic potential, there is an interest for industrial production of this natural compound.

Escherichia coli has been an extensively used host for many industrial applications and is one of the most well characterized model organisms. Furthermore, previous experimental studies by other groups show that curcumin production in E. coli by expression of heterologous enzymes is a feasible process (Katsuyama et al., 2008). One of the problems of using curcumin for therapeutic purposes is that it is rapidly metabolized when taken orally. Therefore, engineering a non-pathogenic E. coli strain for in situ curcumin production can be a promising way to potentiate its pharmacological effects.

The goal of this work consists on the design and optimization of an efficient pathway for curcumin production in $E$. coli. It begins with the identification of the best candidate genes for implementing the heterologous pathway. This pathway will be modeled in silico and inserted into an available kinetic reconstruction of the central carbon metabolism of $E$. coli (Chassagnole et al., 2002). The

\footnotetext{
* Corresponding author. Tel.: +35 1253604414.

E-mail addresses: dmachado@deb.uminho.pt (D. Machado), lrmr@deb.uminho.pt (L.R. Rodrigues), irocha@deb.uminho.pt (I. Rocha).
}

integrated model will then be used for rational design of an optimized strain for curcumin production.

\section{Models and methods}

\subsection{Gene selection}

Curcumin and other curcuminoids are secondary metabolites, that derive from the amino acid metabolism through several enzymatic steps (Fig. 1). Katsuyama and co-workers constructed an artificial pathway for production of curcuminoids in E. coli using the genes of phenylalanine ammonia-lyase (PAL) from the yeast Rhodotorula rubra (step 1), 4-coumarate:CoA ligase (4CL) from Lithospermum erythrorhizon (step 2) and curcuminoid synthase (CUS) from rice (Oryza sativa) (steps 3 and 4) (Katsuyama et al., 2008). It could be observed that direct production of curcuminoids from the metabolic flux of the native metabolism, through phenylalanine and tyrosine, resulted in very low production yields.

In a different experimental setup, carboxylic acids were supplied directly into the medium, which resulted in higher production yields for the different curcuminoids. Therefore, in this work we will assume that the culture medium is supplemented with ferulic acid for the production of curcumin. Hence, only the 3 latter steps are required. Nonetheless, this does not fully decouple curcumin production from the native metabolism, as there is still a requirement of carbon and energy sources, in the forms of malonyl-CoA and ATP, respectively.

In parallel work, the authors identified two enzymes, diketideCoA synthase (DCS) and curcumin synthase (CURS), which are responsible for curcuminoid production in C. longa (Katsuyama 


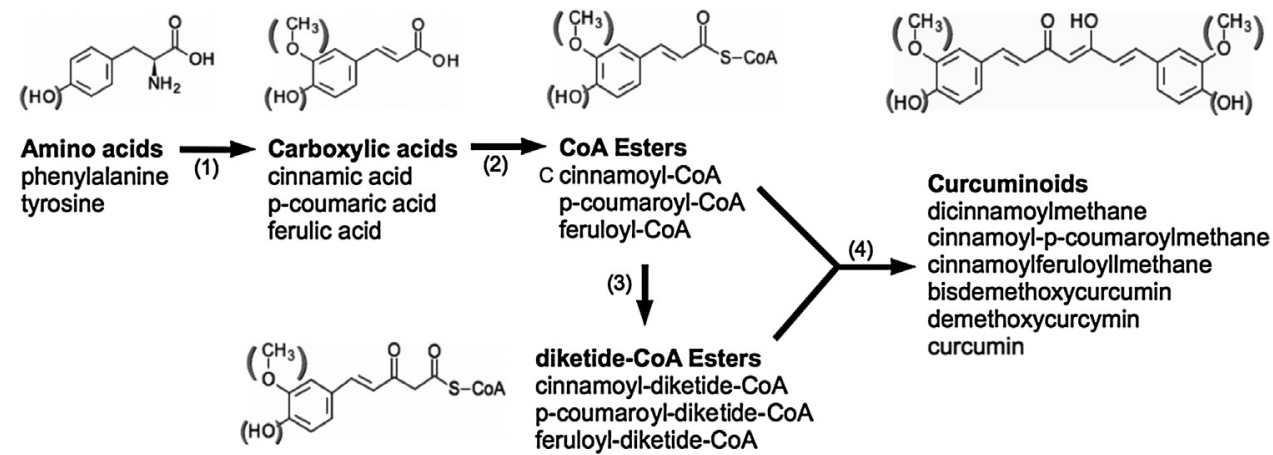

Fig. 1. Curcuminoid production from the amino acid metabolism involves 4 enzymatic steps: (1) deamination, (2) esterification, (3) extension, and (4) condensation.

et al., 2009a). Unlike the CUS enzyme found in rice, DCS and CURS catalyze each of the two steps separately. Also, in vitro enzymatic assays revealed that DCS exhibits allosteric regulation. Together, DCS and CURS represent an efficiently regulated system that prevents depletion of the starter substrate during the condensation phase. Therefore, these two enzymes were chosen for the implementation of the two final steps. For the conversion of ferulic acid into feruloyl-CoA, we selected one of the variants of $4 \mathrm{CL}$ from Arabidopsis thaliana, which have been kinetically characterized (Hamberger and Hahlbrock, 2004). The stoichiometry of the constructed heterologous pathway is the following:

- 4CL: ferulic acid + CoA + ATP $\rightarrow$ feruloyl-CoA + ADP + PPi

- DCS: feruloyl-CoA + malonyl-CoA $+\mathrm{H}^{+} \rightarrow$ feruloyl-diketide-CoA $+\mathrm{CoA}+\mathrm{CO}_{2}$

- CURS: feruloyl-CoA + feruloyl-diketide-CoA $+\mathrm{H}_{2} \mathrm{O} \rightarrow$ curcumin $+\mathrm{CoA}+\mathrm{CO}_{2}$.

\subsection{Kinetic modeling}

We developed a kinetic reconstruction of the curcumin producing mutant strain, by extending a kinetic model of the central carbon metabolism of $E$. coli available in the literature (Chassagnole et al., 2002). The model accounts for the PTS-system, glycolysis and the pentose-phosphate pathway. It also contains analytical rules defining co-metabolite variation after a glucose pulse, which were not used in this work. In order to connect the reconstruction of the heterologous pathway to the native metabolism, the model had to be extended to also include the pathway from pyruvate to malonyl-CoA (Fig. 2).

The kinetic rate laws and kinetic parameters for the new reactions, including the heterologous pathway were instantiated based on information available in the literature. Maximum reaction rate parameters $\left(V_{\max }\right)$ are usually not reported in the literature as they depend on condition-specific enzyme levels. Specific activity values or enzyme turnover numbers $\left(k_{c a t}\right)$ are reported instead. For the heterologous pathway, the concentrations of the respective enzymes were assumed to be in excess $(100 \mathrm{mM})$. For reactions belonging to the native metabolism, the $V_{\max }$ parameters were calculated in the following manner. First, a reference steady-state flux distribution was estimated using a genome-scale model of E. coli (Feist et al., 2007). A Flux Balance Analysis simulation was performed, where the reactions common to both models were constrained to their reference value in the dynamic model $( \pm 0.01 \mathrm{mM} / \mathrm{s})$. Secondly, the parameters were calculated by equalizing the rate laws to the estimated flux $(v)$, and solving for $V_{\max }$, using the same procedure adopted by the authors of the original model:

$v=V_{\max } \cdot f(X, K) \Leftrightarrow V_{\max }=\frac{v}{f(X, K)}$.
In the following, the kinetic reconstruction of each enzymatic step is described in detail. The complete list of kinetic rate laws and parameters is presented in Table 1.

\subsubsection{Pyruvate dehydrogenase (PDH)}

Although PDH was already part of the original model, the concentration of acetyl-CoA was not included in the model, hence the effects of product inhibition were not accounted for. According to Shen and Atkinson (1970) at a concentration of $0.05 \mathrm{mM}$ of acetyl-CoA, the apparent $K_{M}$ value changes from 0.4 to $1.3 \mathrm{mM}$. Assuming a competitive inhibition mechanism, where the apparent $K_{M}$ value is given by the formula $K_{M}^{a p p}=K_{M}\left(1+I / K_{I}\right)$, we obtain $K_{I}=0.0222 \mathrm{mM}$. The rate law was changed to account for product inhibition using this parameter. Note that the $K_{M}$ value from the original model was kept.

\subsubsection{Acetyl-CoA carboxylase (ACCOAC)}

This enzyme catalyzes the carboxylation of acetyl-CoA into malonyl-CoA. The work of Tong (2005) reports a $K_{M}$ value of $0.3 \mu \mathrm{M}$ for accoa. Also, in vitro assays from Freiberg et al. (2004) show that in the opposite direction the binding constant for malonyl-CoA is $100 \mu \mathrm{M}$. Therefore, we adopted a Michaelis-Menten rate law with competitive product inhibition. The $V_{\max }$ parameter was calculated using the method described previously, assuming $1 \mathrm{mM}$ for the steady-state concentrations of acetyl-CoA and malonyl-CoA.

\subsubsection{4-Coumarate:CoA ligase (4CL)}

Kinetic properties of 3 variants of $4 \mathrm{CL}$ for $A$. thaliana were determined by Ehlting et al. (1999). The work presents the specific activity of the 3 variants for different substrates. Another work (Hamberger and Hahlbrock, 2004) reports a 4th variant of this enzyme for the same organism, which has a higher specificity for ferulate $\left(K_{M}=26 \mu \mathrm{M}, S A=153 \mathrm{nmol} / \mathrm{s} / \mathrm{mg}\right)$. We selected this variant, and calculated its turnover number, given its molar mass (62,559 Da).

\subsubsection{Diketide-CoA synthase (DCS)}

This enzyme has been characterized in Katsuyama et al. (2009a). However, the kinetic parameters were estimated using separate equations for each substrate: $k_{\text {cat }}\left(S^{n} /\left(S_{50}^{n}+S^{n}\right)\right)$ for feruloyl-CoA, and $k_{\text {cat }}\left(S /\left(K_{M}+S\right)\right)$ for malonyl-CoA. Since we want one single rate law that takes into account the utilization of both substrates, we recalculated $k_{\text {cat }}$ for the lumped equation given the values in the original article.

\subsubsection{Curcumin synthase (CURS)}

This enzyme has also been kinetically characterized (Katsuyama et al., 2009a,b). From the 3 variants identified, CURS1 was chosen because it has the higher specificity for feruloyl-CoA. The authors have only identified the parameters for the first substrate. 


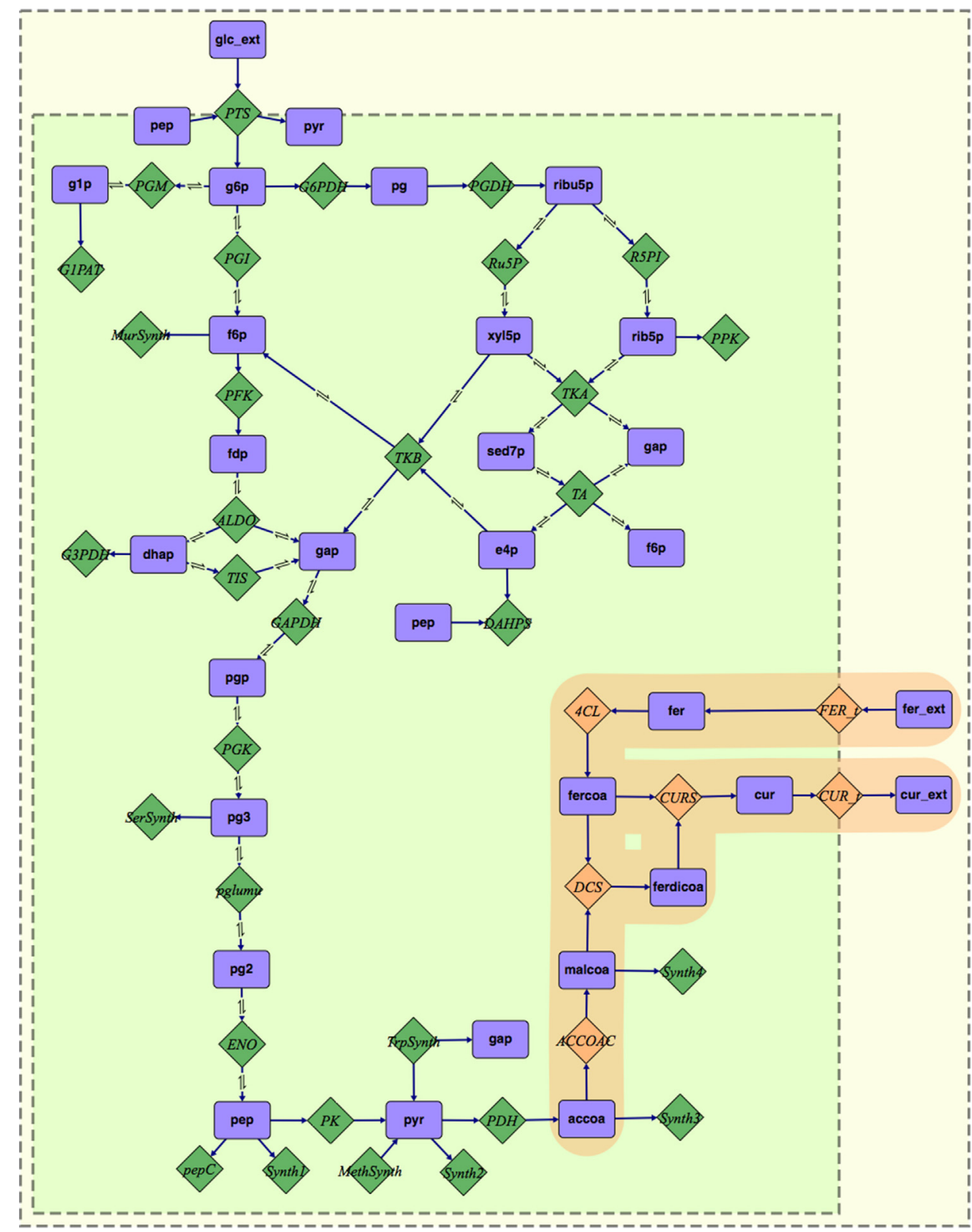

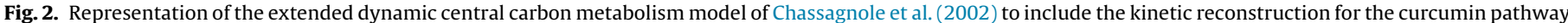

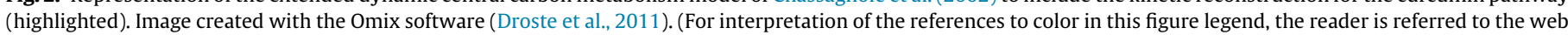
version of the article.)

\section{Table 1}

Kinetic rate laws and respective parameters for the artificial pathway.

\begin{tabular}{|c|c|c|}
\hline Reaction & Kinetic rate law & Parameters \\
\hline PDH & $V_{\max } \frac{p y r^{n}}{p y r^{n}+K_{M}\left(1+\left((a c c o a) /\left(K_{I}\right)\right)\right.}$ & $V_{\max }=270.28 \mathrm{mM} / \mathrm{s}, K_{M}=1159 \mathrm{mM}$ \\
\hline ACCOAC & $V_{\max } \frac{a c c o a}{\operatorname{accoa}+K_{M}\left(1+(\text { malcoa }) /\left(K_{I}\right)\right)}$ & $\begin{array}{l}K_{I}=0.0222 \mathrm{mM}, n=3.68 \\
V_{\max }=0.04634 \mathrm{mM} / \mathrm{s}, K_{M}=0.0003 \mathrm{mM} \\
K_{I}=0.1 \mathrm{mM}\end{array}$ \\
\hline $4 C L$ & $E \cdot k_{\text {cat }} \frac{\text { fercoa }}{K_{M}+\text { fercoa }}$ & $k_{\text {cat }}=9.572 \mathrm{~s}^{-1}, K_{M}=0.026 \mathrm{mM}$ \\
\hline DCS & $E \cdot k_{\text {cat }} \frac{\text { fercoa }^{n}}{K_{M_{1}}^{n}+\text { fercoa }^{n}} \frac{\text { malcoa }}{K_{M_{2}}+\text { malcoa }}$ & $k_{\text {cat }}=0.01343 \mathrm{~s}^{-1}, K_{M_{1}}=0.046 \mathrm{mM}$ \\
\hline CURS & $E \cdot k_{\text {cat }} \frac{\text { fercoa }}{K_{M_{1}}+\text { fercoa }} \frac{\text { ferdicoa }}{K_{M_{2}}+\text { ferdicoa }}$ & $\begin{array}{l}K_{M_{2}}=0.0084 \mathrm{mM}, n=1.8 \\
k_{\text {cat }}=0.02163 \mathrm{~s}^{-1}, K_{M_{1}}=0.018 \mathrm{mM} \\
K_{M}=0.018 \mathrm{mM}\end{array}$ \\
\hline Synth3 & $V_{\max } \frac{a c c o a}{K_{M}+a c c o a}$ & $\begin{array}{l}K_{M_{2}}=0.018 \mathrm{mM} \\
V_{\max }=0.284 \mathrm{mM} / \mathrm{s}, K_{M}=1 \mathrm{mM}\end{array}$ \\
\hline Synth4 & $V_{\max } \frac{\text { malcoa }}{K_{M}+\text { malcoa }}$ & $V_{\max }=0.092372 \mathrm{mM} / \mathrm{s}, K_{M}=1 \mathrm{mM}$ \\
\hline Fer_trans & $k\left(f_{e r} r_{e x t}-\frac{1}{K_{e q}} f e r\right)$ & $k=1000 \mathrm{~s}^{-1}, K_{e q}=1$ \\
\hline Cur_trans & $k\left(\operatorname{cur}-\frac{1}{K_{e q}}\right.$ cur $\left._{\text {ext }}\right)$ & $k=1000 \mathrm{~s}^{-1}, K_{e q}=1$ \\
\hline
\end{tabular}



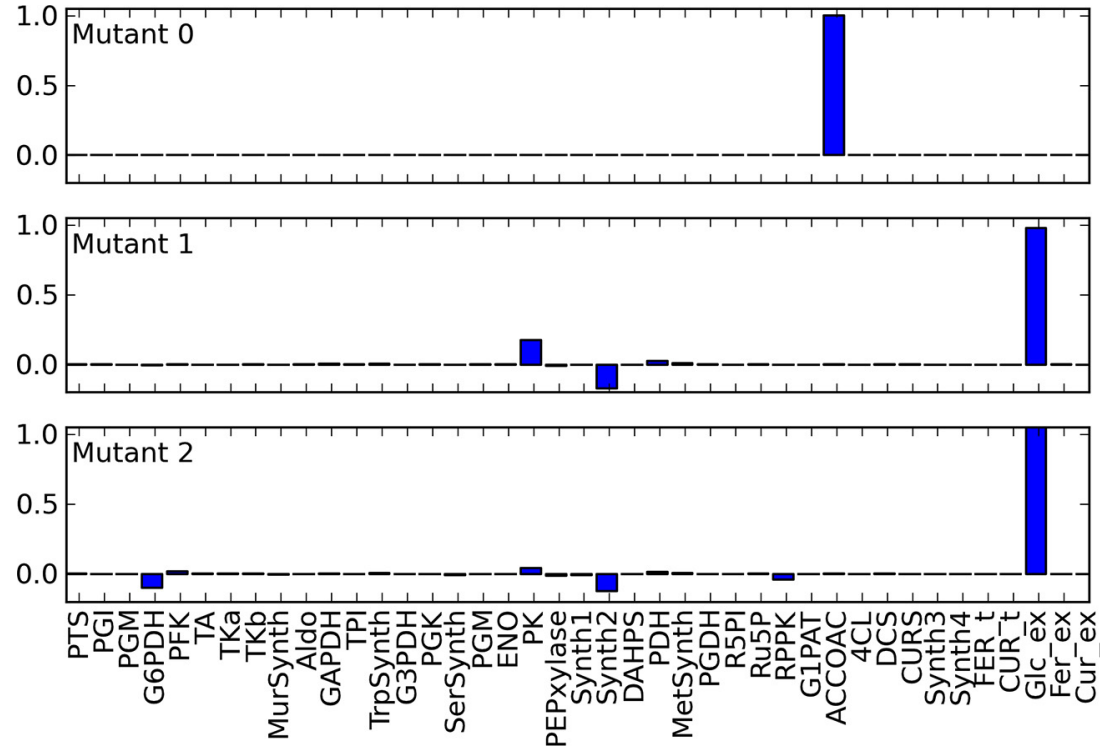

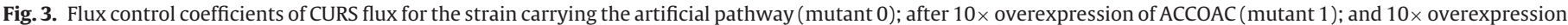
both ACCOAC and PK (mutant2).

Therefore, we assumed that the $K_{M}$ value is the same for both substrates, as the molecules are very similar. Also, as in the previous case, we recalculated $k_{\text {cat }}$ to match the lumped equation that accounts for both substrates.

\subsubsection{Synth3 and Synth4}

These reactions should account for all the flux from acetyl-CoA and malonyl-CoA that is not channeled into the artificial pathway. Following the same strategy used in the original model for Synth1 and Synth2, we assumed Michaelis-Menten kinetics with a $K_{M}$ value of $1 \mathrm{mM}$ for both reactions. The $V_{\max }$ parameters were then estimated using the method described earlier.

\subsubsection{Transport reactions}

From the in vivo experimental work of Katsuyama et al. (2008), we can conclude that $E$. coli must have transport mechanisms for ferulic acid and curcumin. However, since the mechanism is not reported, we assumed reversible mass action kinetics for the rate laws. Also, we assumed that transport is not a rate-limiting step, hence we defined an arbitrarily high value for the kinetic constants.

\section{Results}

The kinetic model developed in this work can be used to perform dynamic simulations, and also to optimize the production of curcumin through the identification of potential bottlenecks. For that matter, we performed metabolic control analysis for the strain carrying the heterologous pathway (mutant 0 ) using the COPASI tool (Hoops et al., 2006). In particular, we calculated the flux control coefficients (FCC) which explain how the change in each individual enzyme affects the whole system, and observed the effect of each reaction over the flux of CURS (Fig. 3).

It can be observed that the rate of ACCOAC is by far the one with higher influence over CURS. Since this influence is positive, we overexpressed the respective enzyme in silico by 10 -fold (mutant 1 ), and recalculated the FCCs. It can now be observed that, for this mutant, the most significant control comes from Glc_ex, the reaction representing the glucose feed. The next enzymatic reaction exerting most control now becomes pyruvate kinase (PK), although with a smaller impact compared to the previous case. We then also overexpressed this enzyme by 10 -fold (mutant 2 ), and again recalculated the FCCs.
By proceeding with this approach, the next logical step is the downregulation of G6PDH, which would decrease the flux through the pentose-phosphate pathway, increasing the flux through glycolysis and, consequently, the availability of acetyl-CoA. However, this can affect biomass production, potentially resulting in a strain with higher yields but lower productivity. This is a trade-off that must be carefully analyzed if such manipulation is to be performed. Furthermore, it can be observed that at this point, glucose supply is the main limiting factor for curcumin production.

Our model does not account for co-metabolite variation. The analytical functions used in the original model for explicit temporal variation are only applicable for the short pulse experiment performed by the authors. Since our fermentation operates in a larger time-scale we used the steady-state concentrations from the original model. We performed sensitivity analysis to evaluate the impact of not explicitly accounting for co-metabolite variation. The sensitivity coefficients of the curcumin production rate with respect to the co-metabolite concentrations were computed and found to fall within the range $\left[-6.48 e^{-6}, 4.94 e^{-5}\right]$. Therefore, we conclude that this assumption does not significantly affect our predictions.

We performed batch simulations of the 3 mutant strains, assuming that ferulic acid is available in excess and that glucose is the limiting substrate (Fig. 4). It can be observed that the overexpression of ACCOAC (mutant 1) significantly increases the production rate of curcumin. The simultaneous overexpression of ACCOAC and PK (mutant 2) does not result in a further increase in the rate of production, yet it increases the expected yield.

\section{Discussion}

Overall, our results show that ACCOAC and PK may be two potential bottlenecks for curcumin production, hence their overexpression is highly recommended, specially the former. In fact, the overexpression of ACCOAC was also performed in Katsuyama et al. (2008) for in vivo curcumin production in E. coli.

It should be noted that the overexpression of PK cannot be excessive, otherwise it can have a negative impact in the whole central carbon metabolism (data not shown). The reason is that an excess of PK, which converts phosphoenol-pyruvate (pep) into pyruvate, can cause a fast depletion of pep, one of the substrates for the 

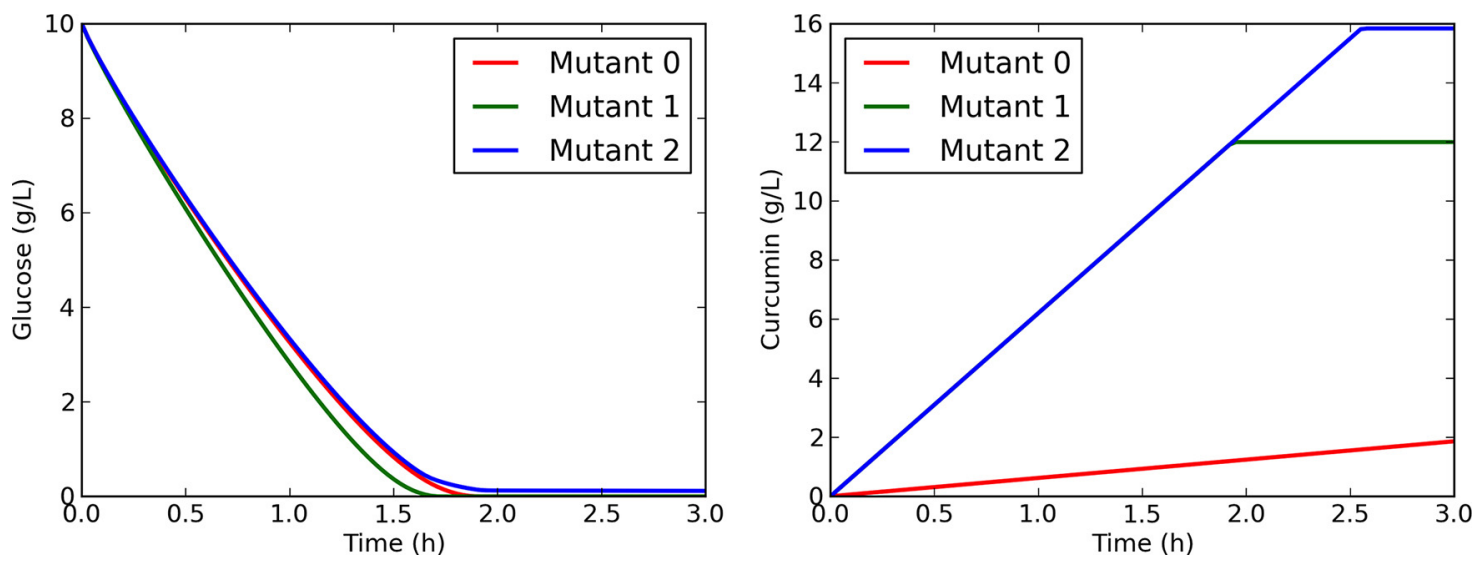

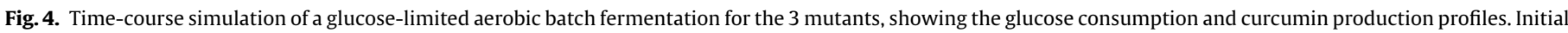
glucose concentration in the reactor was set $10 \mathrm{~g} / \mathrm{L}$, and the fermentations were simulated for $3 \mathrm{~h}$.

phosphotransferase system (PTS). The lack of pep causes a decrease in PTS activity and, consequently, a decrease in glucose uptake.

It is also important to recall our initial assumption that transport is not a rate-limiting step. This assumption was made because transport has been observed but the respective transporters have not been identified. Further experimental work would be necessary to test this assumption. If transport is rate-limiting then the transporters would be the first amplification targets.

As an alternative to MCA, one could have used elementary flux mode (EFM) based methods for rational strain design (Trinh et al., 2008; Hädicke and Klamt, 2011; Soons et al., 2013). This would not require the construction of a kinetic model. However, due to the uncertainty in the flux distribution, EFM-based designs usually require a larger number of manipulations to obtain the desired redirection of the metabolic flux to the target pathway.

Finally, we compared our in silico results to the experimental results of Katsuyama et al. (2008). In their experiment, the authors produced curcumin by supplying ferulic acid to the medium. In this case, glucose was provided in excess $(40 \mathrm{~g} / \mathrm{L})$ and ferulic acid was the limiting substrate $(194 \mathrm{mg} / \mathrm{L})$. This resulted in a production of $113 \mathrm{mg} / \mathrm{L}$ of curcumin, which represents a molar yield of 0.306 . This is bellow the maximum theoretical yield of 0.5 (one molecule of curcumin per two molecules of ferulic acid). We used our model to simulate an experiment in the same conditions, and obtained a total production of $181 \mathrm{mg} / \mathrm{L}$ of curcumin, which represents a molar yield of 0.492 . In this case, the result was independent of the overexpression of glycolytic enzymes, as glucose was not the limiting substrate. We hypothesize that the difference in the yields between the in silico and experimental results is caused by our selection of the DCS/CURS system from C. longa, which exhibits efficient allosteric regulation, whereas the authors selected the multifunctional enzyme CUR from rice for the catalysis of the two final enzymatic steps.

\section{Conclusions}

This work consisted on the design and optimization of a heterologous pathway for curcumin production in E. coli. We developed a kinetic model for the wild-type strain carrying the artificial pathway by extending an already available kinetic model of the central carbon metabolism of E. coli (Chassagnole et al., 2002). Using this model, we performed metabolic control analysis and identified two potential bottlenecks for the production of curcumin when glucose is the limiting substrate. Hence, the overexpression of the respective enzymes is recommended. Simulations performed with the kinetic model further confirm that the proposed manipulations increase the expected curcumin production rates.
The model developed in this work can be used as a framework, not only for rational strain design, but also for implementation of optimal feeding control strategies. Since several assumptions regarding the kinetics of the heterologous pathway were made, it will be necessary to further calibrate the model parameters with experimental data measured from cultivations of the mutant strain. The model has been deposited in the BioModels database (Li et al., 2010) and assigned the identifier MODEL1406060000.

\section{Acknowledgment}

Research supported by the Portuguese Foundation for Science and Technology (FCT), through the project "SYNBIOBACTHER Synthetic biology approaches to engineer therapeutic bacteria" (PTDC/EBB-BIO/102863/2008).

\section{References}

Aggarwal, B., Kumar, A., Bharti, A., et al., 2003. Anticancer potential of curcumin: preclinical and clinical studies. Anticancer Res. 23 (1A), 363-398.

Chassagnole, C., Noisommit-Rizzi, N., Schmid, J., Mauch, K., Reuss, M., 2002. Dynamic modeling of the central carbon metabolism of Escherichia coli. Biotechnol. Bioeng. 79 (1), 53-73.

D’Aguanno, S., D’Agnano, I., De Canio, M., Rossi, C., Bernardini, S., Federici, G., Urbani, A., 2012. Shotgun proteomics and network analysis of neuroblastoma cell lines treated with curcumin. Mol. BioSyst. 8 (4), 1068-1077.

Droste, P., Miebach, S., Niedenführ, S., Wiechert, W., Nöh, K., 2011. Visualizing multi-omics data in metabolic networks with the software omix - a case study. BioSystems 105 (2), 154-161.

Ehlting, J., Büttner, D., Wang, Q., Douglas, C., Somssich, I., Kombrink, E., 1999. Three 4-coumarate: coenzyme a ligases in Arabidopsis thaliana represent two evolutionarily divergent classes in angiosperms. Plant J. 19 (1), 9-20.

Feist, A., Henry, C., Reed, J., Krummenacker, M., Joyce, A., Karp, P., Broadbelt, L., Hatzimanikatis, V., Palsson, B., 2007. A genome-scale metabolic reconstruction for Escherichia coli K-12 MG1655 that accounts for 1260 ORFs and thermodynamic information. Mol. Syst. Biol. 3 (121).

Freiberg, C., Brunner, N., Schiffer, G., Lampe, T., Pohlmann, J., Brands, M., Raabe, M., Häbich, D., Ziegelbauer, K., 2004. Identification and characterization of the first class of potent bacterial acetyl-coa carboxylase inhibitors with antibacteria activity. J. Biol. Chem. 279 (25), 26066-26073.

Hädicke, O., Klamt, S., 2011. Computing complex metabolic intervention strategies using constrained minimal cut sets. Metab. Eng. 13 (2), 204-213.

Hamberger, B., Hahlbrock, K., 2004. The 4-coumarate: Coa ligase gene family in Arabidopsis thaliana comprises one rare, sinapate-activating and three commonly occurring isoenzymes. Proc. Natl. Acad. Sci. U.S.A. 101 (7), 2209.

Hoops, S., Sahle, S., Gauges, R., Lee, C., Pahle, J., Simus, N., Singhal, M., Xu, L., Mendes, P., Kummer, U., 2006. COPASI - a complex pathway simulator. Bioinformatics 22 (24), 3067-3074.

Katsuyama, Y., Kita, T., Funa, N., Horinouchi, S., 2009a. Curcuminoid biosynthesis by two type III polyketide synthases in the herb Curcuma longa. J. Biol. Chem. 284 (17), 11160-11170.

Katsuyama, Y., Kita, T., Horinouchi, S., 2009b. Identification and characterization of multiple curcumin synthases from the Herb Curcuma longa. FEBS Lett. 583 (17), 2799-2803 
Katsuyama, Y., Matsuzawa, M., Funa, N., Horinouchi, S., 2008. Production of curcuminoids by Escherichia coli carrying an artificial biosynthesis pathway. Microbiology 154, 2620-2628.

Li, C., Donizelli, M., Rodriguez, N., Dharuri, H., Endler, L., Chelliah, V., Li, L., He, E., Henry, A., Stefan, M.I., et al., 2010. Biomodels database: an enhanced, curated and annotated resource for published quantitative kinetic models. BMC Syst. Biol. 4 (1), 92.

Palve, Y., Nayak, P., 2012. Curcumin: a wonder anticancer drug. Int. J. Pharm. Biomed. Sci. 3 (2), 60-69.

Shen, L., Atkinson, D., 1970. Regulation of pyruvate dehydrogenase from Escherichia coli. J. Biol. Chem. 245 (22), 5974-5978.
Soons, Z.I., Ferreira, E.C., Patil, K.R., Rocha, I., 2013. Identification of metabolic engineering targets through analysis of optimal and sub-optimal routes. PLOS ONE 8 (4), e61648.

Tong, L., 2005. Acetyl-coenzyme a carboxylase: crucial metabolic enzyme and attractive target for drug discovery. Cell. Mol. Life Sci. 62 (16), 1784-1803.

Trinh, C., Unrean, P., Srienc, F., 2008. Minimal Escherichia coli cell for the most efficient production of ethanol from hexoses and pentoses. Appl. Environ. Microbiol. 74 (12), 3634-3643. 